\title{
Open lung biopsy for investigation of acute respiratory episodes in patients with HIV infection and AIDS
}

\author{
Robert F Miller, Wilfred B Pugsley, Meryl H Griffiths
}

\begin{abstract}
Background-Open lung biopsy (OLB) is rarely necessary for investigation of HIV positive patients with acute respiratory episodes because of the high yield from fibreoptic bronchoscopy with bronchoalveolar lavage (BAL).

Methods-A retrospective review of OLB in HIV positive patients admitted to a specialist inpatient unit with acute respiratory symptoms was carried out in order to define clinical indications, diagnostic yield, impact on management, complications and outcome.
\end{abstract}

Results-OLB was performed in 23 patients; 21 had undergone one or more bronchoscopies with BAL (5 also had negative results from transbronchial biopsy). Indications for OLB were: Group A, 15 patients thought clinically to have pneumocystis pneumonia but not responding to treatment; Group B, 4 patients with focal chest radiographic abnormalities; Group C, 4 patients with diffuse radiographic abnormalities and miscellaneous conditions. Preoperative $\mathrm{PaO}_{2}$ (on air) ranged from 4.4 to 14.5 (mean $=9.5) \mathrm{kPa}$. The results of OLB were in Group A 5 patients had non specific interstitial pneumonitis (NIP), 1 also had Kaposi's sarcoma, 4 had pneumocystis pneumonia (1 also had bronchiolitis obliterans organising pneumonia [BOOP]), 3 had Kaposi's sarcoma and 1 had BOOP and emphysema, 1 had pulmonary infarction and no infection and 1 had normal lung tissue. In Group B diagnoses were NIP, B cell lymphoma, occult alveolar haemorrhage and Pseudomonas aeruginosa pneumonia with BOOP; In Group C 2 patients had NIP and 2 had pneumocystis pneumonia (1 also had cytomegalovirus pneumonitis). All patients survived surgery and none required mechanical ventilation. OLB results significantly affected management; in Group A inappropriate treatment was discontinued in 11 patients found not to have pneumocystis pneumonia, and alternative therapy was begun in the 4 with pneumocystis and in Groups B and $C 6$ patients began specific therapy; unnecessary therapy was avoided in one and antimicrobial treatment was modified in one.

Conclusions-Open lung biopsy in HIV positive patients with focal and diffuse radiographic abnormalities has a high diagnostic yield and low morbidity. This investigation should be considered in those with acute respiratory episodes and negative results from bronchoscopic investigations or who have contra-indications to this procedure.

(Genitourin Med 1995;71:280-285)

Keywords: HIV; AIDS; lung biopsy

Introduction

Before the onset of the AIDS pandemic open lung biopsy was well established as a diagnostic tool for investigation of immunosuppressed patients with diffuse or focal pulmonary infiltrates. ${ }^{1-3}$ Early in the AIDS pandemic open lung biopsy was also frequently performed in order to evaluate respiratory symptoms. ${ }^{4}$ Subsequently the perceived poor prognosis of patients undergoing biopsy, together with the realisation that diagnosis could frequently be made by fibreoptic bronchoscopy with bronchoalveolar lavage with or without transbronchial biopsy ${ }^{5-8}$ meant that open lung biopsy was rarely carried out.

Despite the high yield from bronchoalveolar lavage and transbronchial biopsy occasionally HIV positive patients with symptoms and signs of lower respiratory tract disease have negative results from these investigations and open lung biopsy is necessary to provide a diagnosis and enable specific treatment to be given. In this study we describe the clinical indications, diagnostic yield and impact on management, complications and outcome of open lung biopsy in HIV positive patients who had acute respiratory episodes, many of whom had negative bronchoscopic investigations.

\section{Methods}

Between October 1987 and March 1994754 HIV-1 antibody positive patients were admitted to a specialist inpatient unit for investigation of acute respiratory episodes and were under the care of a respiratory physician. During this period $23 \mathrm{HIV}$ antibody positive patients underwent open lung biopsy. In each case we recorded the clinical indications for biopsy, the type and number of any investigations performed and treatment given before biopsy, arterial blood gases (on air), the diagnosis obtained and impact on management, and complications arising from open lung biopsy. 
In those who underwent fiberoptic bronchoscopy before open lung biopsy the technique was performed as previously described, ${ }^{79}$ and bronchoalveolar lavage fluid routinely stained and cultured for bacteria, fungi, mycobacteria and viruses. Transbronchial biopsy specimens were taken without fluoroscopic control; four or more specimens being obtained on each occasion. Biopsies were stained and cultured as for bronchoalveolar lavage fluid. ${ }^{79}$

Twenty two patients were male (21 were homosexual Caucasians and one was a heterosexual African) and the one female was a heterosexual Caucasian. Their ages ranged from 24 to 56 (mean $42 \cdot 5$ ) years. All had normal renal function, platelet count and clotting screen. Open lung biopsy was performed using the technique described by Venn et al. ${ }^{10}$ In summary surgery was performed via an anterolateral mini-thoracotomy, on the right if there was bilateral symmetrical disease radiographically, otherwise on the side of maximum radiographic abnormality. A representative portion of abnormal lung was taken. A chest drain was routinely left in place following closure, placed on $2 \mathrm{kPa}$ suction and removed at 24 to 48 hours. Each sample of lung tissue was split and part was processed for histology, the remainder was cultured to identify bacteria, fungi, mycobacteria and viruses.

\section{Results}

The clinical indications for open lung biopsy were; Group A, 15 patients who were thought clinically to have Pneumocystis carinii pneumonia. All had presented with typical symptoms

Investgations, treatment and diagnosis in patients undergoing open lung biopsy

\begin{tabular}{|c|c|c|c|c|}
\hline Group/No & $\begin{array}{l}\text { Negative investigations } \\
\text { prior to open lung biopsy }\end{array}$ & $\begin{array}{l}\text { Treatment prior to } \\
\text { open lung biopsy }\end{array}$ & $\begin{array}{l}\mathrm{PaO}_{2} \\
(\mathrm{kPa})\end{array}$ & Final diagnosis \\
\hline \multicolumn{5}{|l|}{ Group A } \\
\hline & $\mathrm{BAL}(\times 2)$ & IV co-trimoxazole & $8 \cdot 7$ & Normal lung \\
\hline 2 & $\mathrm{BAL}+\mathrm{TBB}(\times 2)$ & IV pentamidine & $11 \cdot 2$ & Kaposi's sarcoma \\
\hline 3 & BAL $(\times 2)$ & Neb pentamidine & $10 \cdot 1$ & BOOP + emphysema \\
\hline 4 & IS, BAL, BAL + TBB & IV co-trimoxazole & $9 \cdot 5$ & Granulomatous PCP \\
\hline 5 & IS $(\times 2), \mathrm{BAL}(\times 3)$ & Neb pentamidine & $13 \cdot 9$ & Granulomatous PCP \\
\hline 6 & IS, BAL & Neb pentamidine & $8 \cdot 0$ & $\mathrm{PCP}+\mathrm{BOOP}$ \\
\hline 7 & IS, BAL $(\times 3)$ & IV co-trimoxazole & $9 \cdot 9$ & NIP \\
\hline 8 & IS, BAL $(\times 2)$ & Neb pentamidine & $9 \cdot 1$ & Granulomatous PCP \\
\hline 9 & BAL & IV co-trimoxazole & $8 \cdot 7$ & Kaposi's sarcoma \\
\hline 10 & $\mathrm{BAL}+\mathrm{TBB}$ & IV co-trimoxazole & $4 \cdot 4$ & NIP \\
\hline 11 & BAL & IV co-trimoxazole & $11 \cdot 4$ & NIP \\
\hline 12 & BAL $(\times 2)$ & IV co-trimoxazole & $6 \cdot 0$ & NIP + Kaposi's sarcoma \\
\hline 13 & $\mathrm{BAL}(\times 2)$ & IV pentamidine & $9 \cdot 9$ & Pulmonary infarction \\
\hline 14 & BAL & IV co-trimoxazole & $9 \cdot 9$ & Kaposi's sarcoma \\
\hline 15 & BAL & IV co-trimoxazole & $6 \cdot 7$ & NIP \\
\hline \multicolumn{5}{|l|}{ Group B } \\
\hline 16 & BAL & - & $10 \cdot 7$ & $\begin{array}{c}\text { Non-Hodgkin's } \\
\text { lymphoma }\end{array}$ \\
\hline 17 & BAL & - & $10 \cdot 3$ & Occult alveolar \\
\hline 18 & $\mathrm{BAL}, \mathrm{BAL}+\mathrm{TBB}$ & - & $14 \cdot 5$ & NIP \\
\hline 19 & BAL $(\times 2)$ & IV cefuroxime & $11 \cdot 6$ & $\begin{array}{r}\text { Pseudomonas aeruginosa } \\
\text { pneumonia + BOOP }\end{array}$ \\
\hline \multicolumn{5}{|l|}{ Group C } \\
\hline 20 & $\mathrm{BAL}+\mathrm{TBB}$ & - & ND & NIP \\
\hline 21 & BAL & - & $9 \cdot 1$ & NIP \\
\hline 22 & NIL & IV co-trimoxazole & $6 \cdot 3$ & $\mathrm{PCP}+\mathrm{CMV}$ \\
\hline 23 & NIL & IV pentamidine & $8 \cdot 9$ & Granulomatous PCP \\
\hline
\end{tabular}

$\mathrm{BAL}=$ Bronchoalveolar lavage, $\mathrm{TBB}=$ Transbronchial biopsy, IS = Induced sputum, ND = not done, BOOP = Bronchiolitis obliterans organizing pneumonia, NIP = Non specific interstitial pneumonitis, $\mathrm{PCP}=$ Pneumocystis carinii pneumonia, $\mathrm{CMV}=$ cytomegalovirus, $\mathrm{Neb}=$ Nebulised, IV = Intravenous. of non-productive cough, increasing exertional dyspnoea and fever. ${ }^{11}$ Chest radiographs were abnormal showing diffuse infiltrates ranging in severity from subtle perihilar changes to more marked bilateral interstitial shadowing; patients were variably hypoxic. CD4 lymphocyte cell counts in these patients ranged from 10 to 260 (median $60) / \mathrm{mm} .^{3}$ Despite specific anti-pneumocystis therapy, patients were deteriorating with worsening dyspnoea, falling arterial oxygen tensions $\left(\mathrm{PaO}_{2}\right)$, and many had increasing chest radiographic abnormalities (table); Group B, four patients who had fever and focal radiographic abnormalities. A diagnosis of non Hodgkin's lymphoma had been made, following lymph node and bone marrow biopsy, six months previously in patient 17 . Patient 19 failed to respond to IV cefuroxime for an Hemophilus influenzae infection diagnosed at bronchoalveolar lavage; Group C, four patients with specific clinical problems. Patient 20 had extensive cutaneous Kaposi's sarcoma. He presented with dyspnoea and a chest radiograph showed diffuse bilateral interstitial infiltrates. He was thought clinically to have pulmonary Kaposi's sarcoma, for which chemotherapy was planned. Patient 21 had no AIDS defining illness, a CD4 count of 440 (normal $350-2200$ ) $/ \mathrm{mm}^{3}$ and a lymphocytic bronchoalveolar lavage sample, having presented with 12 weeks progressive dyspnoea and diffuse bilateral radiographic infiltrates. He was thought clinically not to have pneumocystis pneumonia, but sarcoidosis or a lymphoproliferative disorder were suggested by the bronchoalveolar lavage findings. Patient 22, a Caucasian female had presented with a severe pneumonia and was treated for presumed pneumocystis pneumonia with IV co-trimoxazole and methylprednisolone. Mechanical ventilation was required initially. Following an initial recovery, with improvement in $\mathrm{PaO}_{2}$ and extubation, there was subsequent deterioration with worsening hypoxia and radiographic abnormalities. The final patient (23) presented with a pneumothorax, thus bronchoscopy was contra-indicated. The chest radiograph showed in addition, bilateral apical bullae and diffuse interstitial shadowing. Intercostal tube drainage of the pneumothorax was unsuccessful because of persistent air leak through the bullae. Surgical bullectomy and pleuradesis were carried out together with open lung biopsy.

Results of investigations performed with negative results and treatment received before open lung biopsy, arterial blood gases immediately before biopsy and the diagnosis provided by histological and microbiological analysis of lung tissue are shown in the table. It may be noted that no patient was mechanically ventilated at the time of biopsy and only patients 10, 12, 15 and 22 had received steroids.

Open lung biopsy provided diagnostic histological material in all patients. The results of open lung biopsy had a significant impact on management. In Group $A$ in the 11 patients without Pneumocystis carinii pneumonia 
unnecessary and potentially toxic treatment was stopped and in addition the four patients with pulmonary Kaposi's sarcoma (including the one who also had non specific interstitial pneumonitis) began chemotherapy with vincristine and bleomycin, the patient with bronchiolitis obliterans organising pneumonia (BOOP) and paraseptal emphysema began prednisolone, as did the five patients with non specific interstitial pneumonitis. Three of the four patients with $P$ carinii pneumonia had been treated with nebulised pentamidine. This therapy was stopped and IV co-trimoxazole started in two patients (the third, who was allergic to co-trimoxazole, was given IV pentamidine). In the fourth patient, who was already receiving IV co-trimoxazole, therapy was continued unchanged. In Group B patient 16 with non Hodgkin's lymphoma began chemotherapy, patient 17 with occult alveolar haemorrhage avoided antibiotics, patient 18 with non specific interstitial pneumonitis began prednisolone and the patient with Pseudomonas aeruginosa and BOOP discontinued cefuroxime and began ceftazidime. In Group C patient 20 avoided chemotherapy and instead received corticosteroids. This therapy was also given to patient 21 . In patient 22 IV ganciclovir was added as treat ment for cytomegalovirus pneumonitis and co-trimoxazole continued for the confirmed diagnosis of pneumocystis pneumonia? Patient 23 was given IV pentamidine (bein allergic to co-trimoxazole).

All patients survived open lung biopsy an despite some patients being profoundly, hypoxaemic none required post-operative mechanical ventilation. Lower respirator tract infection requiring physiotherapy and antibiotics occurred post operatively in two patients, one of whom also had a persistent ai $\vec{w}$ leak and so the chest drain was left in place oे
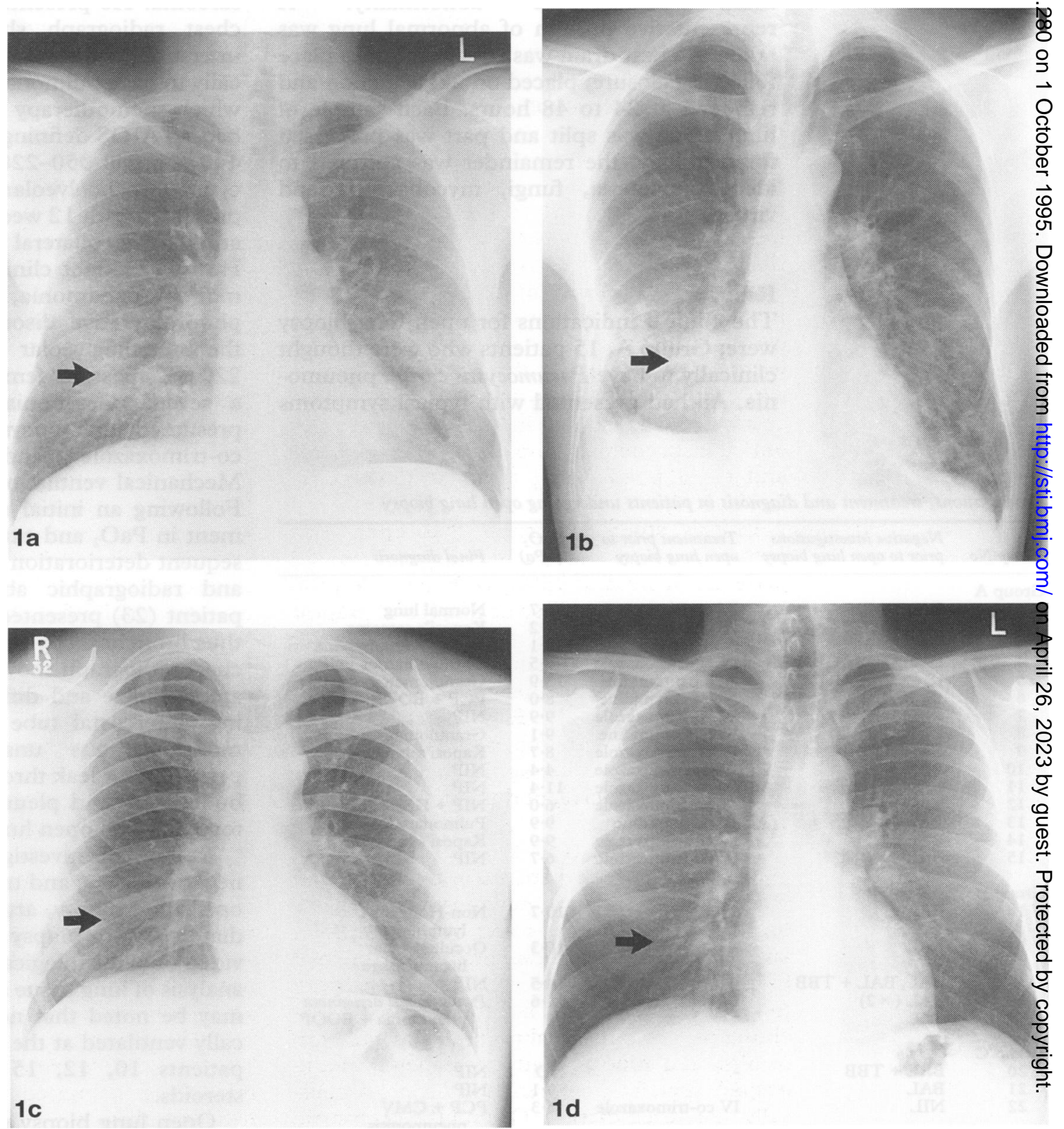

(a) Chest radiograph of Patient 4: Diagnosis = Granulomatous Pneumocystis carinii pneumonia. The arrow indicates the site of biopsy. (b) Chest radiograph of Patient 14: Diagnosis = Pulmonary Kaposi's sarcoma. The arrow indicates the site of biopsy. (c) Chest radiograph of Patient 15: Diagnosis = Non specific Interstitial Pneumonitis. The arrow indicates the site of biopsy. (d) Chest radiograph of Patient 21: Diagnosis = Non specific Interstitial Pneumonitis. The arrow indicates the site of biopsy. 
on suction, for 72 hours. Two other patients had superficial wound infections not requiring wound toilet but responding to antibiotics. None of the patients with postoperative infections had received steroids. Subcutaneous emphysema or hydrothorax did not occur in any patient. All but two patients were eventually discharged from hospital; patients 12 and 13 died 42 and 69 days respectively after biopsy, from the effects of progressive HIV disease. Examples of chest radiographic abnormalities and the site of open lung biopsy are shown in the figure.

\section{Discussion}

We have previously undertaken a prospective study comparing the value of transbronchial biopsy and bronchoalveolar lavage in the diagnosis of pulmonary disease in HIV infected individuals with respiratory episodes. ${ }^{7} \mathrm{We}$ found that bronchoalveolar alveolar lavage was superior to transbronchial biopsy for diagnosis of $P$ carinii pneumonia (90 vs $56 \%$ ) cytomegalovirus infection (100 vs $33 \%$ ) and other pathogens. The diagnosis of pulmonary Kaposi's sarcoma was not made by either bronchoalveolar lavage or transbronchial biopsy. In no case was the diagnosis missed by lavage but made by transbronchial biopsy. We found a complication rate of $22 \%$ in those investigated with transbronchial biopsy, including pneumothorax and profuse haemorrhage (fatal in one patient); complications were especially likely in those with $P$ carinii pneumonia. In contrast there were no significant complications in patients investigated with lavage alone. ${ }^{7}$ On the basis of this study and data from others, ${ }^{58}$ we perceived no cost/benefit advantage in continuing to carry out transbronchial biopsies in our patients and so stopped doing them at the end of 1989.

In this study we found that open lung biopsy produced a specific diagnosis in 22 patients, (a yield of $95 \%$ ), with acute respiratory episodes and diffuse or focal radiographic abnormalities, most of whom had negative bronchoscopic investigations. These results are in keeping with other studies in patients immunosuppressed by HIV infection ${ }^{4}$ or by other causes, ${ }^{1-3}$ with reported yield in those with diffuse radiographic infiltrates of between 83 and $95 \%$, and up to $71 \%$ in those with focal infiltrates. ${ }^{14}$

In a study from New York by Fitzgerald $e t$ al, $42 \mathrm{HIV}$ positive patients underwent open lung biopsy, 29 had previously undergone fibreoptic bronchoscopy and bronchoalveolar lavage (18 had also undergone transbronchial biopsy) with negative results, four were thought clinically to be too ill for bronchoscopy or had abnormal coagulation results, and nine patients were deteriorating despite specific treatment for bronchoscopically diagnosed disease. Overall the diagnostic yield was $95 \%$, in those with negative bronchoscopic investigations the commonest diagnoses were Pneumocystis carinii pneumonia in nine patients, Kaposi's sarcoma in seven, interstitial fibrosis without infection in five and Mycobacterium tuberculosis in two patients; a specific diagnosis was obtained in all four who had not undergone brónchoscopy, including Kaposi's sarcoma in one case, but in those deteriorating despite a confirmed diagnosis, new diagnostic information requiring a change of treatment was obtained in only two patients. ${ }^{4}$

In our study the finding of Kaposi's sarcoma at open lung biopsy in four patients with diffuse radiographic infiltrates thought clinically to have $P$ carinii pneumonia was not surprising as pulmonary Kaposi's sarcoma has been described mimicking this condition. ${ }^{12}{ }^{13}$ Parenchymal lung involvement by Kaposi's sarcoma may also occur even if tracheobronchial disease is not evident at bronchoscopy. ${ }^{12}$ The diagnosis of lung parenchymal Kaposi's sarcoma may be missed by transbronchial biopsy. This may be due to the patchy nature of parenchymal infiltration, together with the fact that lesions rarely show cytological features of malignancy and biopsy specimen crush artefact, haemorrhage and granulation tissue appearances may all mimic Kaposi's sarcoma. ${ }^{1214}$ In one study, where transbronchial biopsy was routinely performed for diagnosis, the yield from this technique for pulmonary Kaposi's sarcoma was only $23 \% .^{14}$ The diagnosis of Kaposi's sarcoma was missed by transbronchial biopsy in the patient in our study, and in seven of the eight patients with this diagnosis in Fitzgerald's study. ${ }^{4}$ These data suggest that the need for open lung biopsy would not have been obviated in our patients with pulmonary Kaposi's sarcoma even if we had routinely performed transbronchial biopsy at bronchoscopy. Occult alveolar haemorrhage, found in one of our patients at open lung biopsy was once thought to be specific for the diagnosis of pulmonary Kaposi's sarcoma, ${ }^{13}$ but is now known to be a non specific finding in HIV positive patients with respiratory symptoms, ${ }^{15}$ and is also seen in $P$ carinii pneumonia, bacterial and mycobacterial infection.

Four of our patients received nebulised pentamidine. This therapy was formerly very fashionable for the treatment of mild to moderate severity $P$ carinii pneumonia. ${ }^{16}$ It is now known that patients receiving this form of therapy take longer to respond to treatment, compared with patients treated with IV cotrimoxazole; arterial blood gases and chest radiographs may worsen during the first 10-14 days of treatment, before eventual recovery. ${ }^{16}$ There is also a higher rate of early relapse following successful treatment and as treatment is not systemically delivered it may not suppress extra pulmonary pneumocystosis. For these reasons nebulised pentamidine is rarely, if ever, used now for treatment. If we had used IV co-trimoxazole or pentamidine instead of nebulised pentamidine in these patients then we might have anticipated recovery, without need for open lung biopsy, in the three who had $P$ carinii pneumonia. The histological variant form granulomatous $P$ pneumonia, diagnosed in four patients in 
our study is well described in HIV positive patients. The diagnosis cannot be made by conventional histochemical staining of bronchoalveolar lavage fluid as the organisms do not lie within alveoli, instead they are "walled off" by palisades of histiocytes. The granulomatous response is patchy and transbronchial biopsy may also be negative. ${ }^{17}$ Molecular biological techniques used to identify $P$ carinii by DNA amplification using the polymerase chain reaction with $P$ carinii-specific oligonucleotide primers is still largely a research tool. ${ }^{18}$ Prospective comparisons have shown DNA amplification to be superior to conventional silver staining for detection of $P$ carini in bronchoalveolar lavage fluid and induced sputum. ${ }^{1920}$ More recently the technique has been successfully applied to patients with histologically variant granulomatous $P$ carinii pneumonia. Here, although silver staining of bronchoalveolar alveolar lavage fluid was negative and the diagnosis was made by open lung biopsy, retrospective analysis of lavage fluid by DNA amplification confirmed the diagnosis. ${ }^{21}$ With future refinements and appropriate calibration this technique may have a clinical role in diagnosis of $P$ carini pneumonia, in particular patients with histologically atypical disease, and further reduce the need for open biopsy to confirm the diagnosis.

Non specific interstitial pneumonitis, which may clinically and radiographically simulate $P$ carinii pneumonia, ${ }^{22}$ has been identified in up to $38 \%$ of HIV positive patients undergoing bronchoscopy for evaluation of respiratory symptoms. ${ }^{23}$ It is perhaps surprising that as the diagnosis can be made by transbronchial biopsy, results from this investigation were negative in the three patients in our study who underwent this procedure.

An alternative strategy in those patients with focal radiographic abnormalities might have been to use percutaneous lung biopsy under fluoroscopic or CT guidance. However, this procedure carries the risk of pneumothorax and uncontrolled bleeding and there are reports of cardiorespiratory arrest associated with the technique. ${ }^{24}$ In contrast open lung biopsy affords the opportunity to view the lung directly and identify an abnormal area before obtaining tissue and larger samples may be obtained for analysis. Haemostasis can readily be achieved and the surgical pleural leak carefully closed: an intercostal drain placed on suction prevents pneumothorax.

The technique of open lung biopsy in our patients was safe. The complication rate was surprisingly low with minor wound infections in only two patients and chest infections in two others. These data are in contrast to other reports where wound infection and poor healing have been commoner. ${ }^{1}{ }^{25}$ In these studies a higher proportion of patients had received glucocorticoids, a factor that might have encouraged infection or delayed healing. Of note none of our patients who had received steroids developed infections.

Cardiothoracic surgeons in other centres may be asked by physician colleagues to perform open lung biopsy on HIV positive patients. They may question the usefulness of the procedure in terms of the risks and likely benefits to the patient. Our data, showing a high yield and low morbidity, support the use of this technique in selected patients. Based on these data our unit policy is now to proceed to open lung biopsy in patients with focal and diffuse radiographic abnormalities only if there are negative results from one or more fibreoptic bronchoscopy (with bronchoalveolar lavage) or if a patient has contraindications to bronchoscopy. In addition, in those with diffuse radiographic abnormalities, we would only consider open lung biopsy if there was clinical and/or radiographic deterioration despite specific anti-pneumocystis therapy.

In conclusion, open lung biopsy in HIV positive patients with symptoms and signs of lower respiratory tract disease and focal or diffuse radiographic abnormalities, with either negative results from bronchoscopic investigations or who have contra indications to the procedure, has a high diagnostic yield and results have a significant impact on management, enabling specific therapy to be given and unnecessary potentially toxic treatment to be avoided. The procedure is safe and has low morbidity.

We thank Jane Healing and Ursuline Loubser for typing the manuscript.

1 Jaffe JP, Maki DG. Lung biopsy in immunocompromised patients; one institution's experience and an approach to management of pulmonary disease in the compromised host. Cancer 1981;48:1144-53.

2 Michaelis LL, Leight GS, Powell RD, DeVita VT. Pneumocystis pneumonia: the importance of early lung biopsy. Ann Surg 1976;183:301-6.

3 Rosen PP, Martini N, Armstrong D. Pneumocystis carini pneumonia. Diagnosis by lung biopsy. Am $f \mathrm{Med}$ 1975;58:794-801

4 Fitzgerald W, Bevelaqua FA, Garay SM, Aranda CP. Th role of open lung biopsy in patients with the acquired immunodeficiency syndrome. Chest 1987;91:659-61.

5 Broaddus C, Dake MD, Stulbarg MS, et al. Bronchoalveolar lavage and transbronchial biopsy for the diagnosis of pulmonary infections in the acquired immunodeficiency syndrome. Ann Intern Med 1985; 102:747-52.

6 Blumenfeld W, Wager E, Hadley WK. Use of the transbronchial biopsy for diagnosis of opportunistic pulmonary infections in acquired immunodeficiency syndrome (AIDS). Am f Clin Pathol 1984;81:1-5

7 Griffiths MH, Kocjan G, Miller RF, Godfrey-Faussett P. Diagnosis of pulmonary disease in human immunodeficiency virus infection: role of transbronchial biopsy and bronchoalveolar lavage. Thorax 1989;44:554-8.

8 Orenstein M, Webber CA, Cash M, Henrich AE. Value of bronchoalveolar lavage in the diagnosis of pulmonary infection in acquired immune deficiency syndrome. Thorax 1986;41:345-9.

9 Miller RF, Kocjan G, Buckland J, Holton J, Malin A Semple SJG. Sputum induction for the diagnosis of pul monary disease in HIV positive patients. $f$ Infection monary disease

10 Venn GE, Kay PH, Midwood CJ, Goldstraw P. Open lung biopsy in patients with diffuse pulmonary shadowing Thorax 1985;40:931-5.

11 Miller RF, Millar AB, Weller IVD, Semple SJG. Empirica treatment without bronchoscopy for Pneumocystis carin pneumonia in the acquired immunodeficiency syndrome. Thorax 1989;44:559-64.

12 Miller RF, Tomlinson MC, Cottrill CP, Donald J, Spittle MF, Semple SJG. Bronchopulmonary Kaposi's sarcom in patients with AIDS. Thorax 1992;47:721-5.

13 Fouret PJ, Touboul JL, Mayaud CM, Akoun GM, Roland J. Pulmonary Kaposi's sarcoma in patients with acquired study. Thorax 1987;42:262-8.

14 Meduri GU, Stover DE, Lee M, Myskowski PL, Carvelli $\mathrm{JF}$, Zaman MB. Pulmonary Kaposi's sarcoma in the acquired immunodeficiency syndrome. Am f Med acquired imm 
15 Hughes-Davies L, Kocjan G, Spittle MF, Miller RF. Occult alveolar haemorrhage in Bronchopulmonary Kaposi's sarcoma. 7 Clin Pathol 1992;45:536-7.

16 Miller RF, Godfrey Fausset P, Semple SJG. Nebulised pentamidine as treatment for Pneumocystis carinii pneumonia in the acquired immunodeficiency syndrome. monia in the acquired

17 Foley NM, Griffiths MH, Miller RF. Histologically atypical Pneumocystis carinii pneumonia. Thorax 1993;48: 996-1001.

18 Wakefield AE, Pixley FJ, Banerji S, et al. Amplification of mitochondrial ribosomal RNA sequences from Pneumocystis carinii DNA of rat and human origin. Mol Biochem Parasitol 1990;43:69-76.

19 Wakefield AE, Pixley FJ, Banerii S, Miller RF, Moxon ER Hopkin JM. Detection of Pneumocystis carinii with DNA amplification. Lancet 1990;336:451-3.

20 Wakefield AE, Guiver LA, Miller RF, Hopkin JM. DNA amplification on induced sputum samples for diagnosis of Pneumocystis carinii pneumonia. Lancet 1991;337: 1378-9.

21 Wakefield AE, Miller RF, Guiver LA, Hopk JM. Granulomatous Pneumocystis carinii pneumonia: DNA amplification studies on bronchoscopic alveolar lavage amplification studies on bronch

22 Simmons JT, Suffredini AF, Lack EE, et al. Nonspecific interstitial pneumonitis in patients with AIDS: radiologic features. $A \Varangle R$ 1987;149:265-8.

23 Suffredini AF, Ognibene FP, Lack EE, et al. Nonspecific interstitial pneumonitis: a common cause of pulmonary disease in the acquired immunodeficiency syndrome. Ann Intern Med 1987;107:7-13.

24 Craddock C, Pasvol G, Ball R, et al. Cardiorespiratory arrest and autonomic neuropathy in AIDS. Lancet 1987;ii:16-8.

25 Greenman RL, Goodall PT, King D. Lung biopsy in immunocompromised hosts. Am $\mathcal{f}$ Med 1975;59: 488-96. 